\title{
Composição Bromatológica, Energia Metabolizável e Equações de Predição da Energia do Grão e de Subprodutos do Trigo para Pintos de Corte ${ }^{1}$
}

\author{
Ricardo Vianna Nunes², Horacio Santiago Rostagno ${ }^{3}$, Luiz Fernando Teixeira Albino³, \\ Paulo Cezar Gomes 3 , Rodrigo Santana Toledo ${ }^{4}$
}

\begin{abstract}
RESUMO - Foram determinados os valores de energia metabolizável aparente (EMA) e energia metabolizável aparente corrigida pelo balanço de nitrogênio $\left(\right.$ EMA $_{n}$ ) e elaboradas equações de predição dos valores energéticos, utilizando a composição química de 11 alimentos. Os alimentos avaliados foram: farinha morena, farinha de trigo, trigo-grão, triguilho, gérmen de trigo, resíduo de biscoito, resíduo de macarrão e quatro farelos de trigo. Foi utilizado o método tradicional de coleta total de excretas, com 480 pintos de corte, de 16 a 24 dias de idade. O delineamento experimental foi o inteiramente casualizado, com 11 alimentos e uma ração-referência, quatro repetições e 10 aves por unidade experimental (cinco machos e cinco fêmeas). Cada alimento substituiu a ração-referência em 40\%. Os valores de EMA e EMA $_{\mathrm{n}}$, expressos em kcal/kg de matéria seca (MS), dos alimentos variaram de 1807 a 1758 para o farelo de trigo 3 e de 4480 a 4339 para o resíduo de biscoito, respectivamente. As equações de predição que melhor estimaram os valores de EMA e EMA $\left(\mathrm{R}^{2}=98 \%\right)$ foram aquelas em que foram usados os conteúdos de proteína bruta (PB) e, ou, fibra em detergente neutro (FDN), com $\mathrm{R}^{2}$ igual a 98\%, sendo: EMA (kcal/kg MS $)=4910,03-47,82 * \mathrm{~PB}-47,77 * F D N ;$ e EMA $(\mathrm{kcal} / \mathrm{kg}$ MS $)=4754,02-48,38 * \mathrm{~PB}-45,32 * \mathrm{FDN}$
\end{abstract}

Palavras-chave: energia metabolizável, equações de predição, pintos de corte

\section{Chemical Composition, Metabolizable Energy and Energy Prediction Equations of Wheat Grain and Wheat By-Products for Broiler Chicks}

\begin{abstract}
The apparent metabolizable energy (AME) and nitrogen corrected apparent metabolizable energy (AME ( values were determined and energy prediction equations obtained using the chemical composition of 11 feedstuffs. The feedstuffs evaluated were: brown flour meal, wheat flour, wheat grain, wheat grain residue, wheat germ, cookies residue, macaroni residue and, four wheat brans. Total excreta collection method was used, with 480 broiler chicks, 16 to 24 days old. A randomized complete design was utilized, with 11 feedstuffs and a basal reference diet, using four replications and ten birds per experimental unit (five males and five females). Each feedstuff replaced the reference diet at the amount of $40 \%$. The feedstuffs AME and AME $\mathrm{A}_{\mathrm{n}}$ alues expressed in kcal/kg of dry matter (DM), varied from 1807 to 1758 for wheat bran 3 and from 4480 to 4339 for cookies residue, respectively. The prediction equations that best estimated $\left(\mathrm{R}^{2}=98 \%\right)$ AME and $A M E_{n}$ values were those in which crude protein $(\mathrm{CP})$ and/or neutral detergent fiber (NDF) contents, were used AME $(\mathrm{kcal} / \mathrm{kg} \mathrm{DM})=4910,03-47,82 * \mathrm{CP}-47,77 * \mathrm{NDF}$; and AME ${ }_{\mathrm{n}}$ $(\mathrm{kcal} / \mathrm{kg} \mathrm{DM})=4754.02-48.38 * \mathrm{CP}-45.32 * \mathrm{NDF}$.
\end{abstract}

Key Words: broilers chicks, metabolizable energy, prediction equations

\section{Introdução}

A formulação e o balanceamento de rações consistem na mistura de vários alimentos, com a finalidade de atender as exigências nutricionais dos animais, para que possam expressar o máximo de seu potencial genético. Torna-se necessário, então, conhecer a composição nutricional e os respectivos valores energéticos dos alimentos, bem como suas limitações nutricionais.

A grande variação na composição dos alimentos disponíveis no Brasil, devido a diversos fatores, é um grande problema enfrentado pelos nutricionistas.
Assim, vários trabalhos têm sido desenvolvidos com o objetivo de atualizar os valores nutricionais dos alimentos comumente utilizados nas rações de aves e suínos e, também, conhecer o valor nutritivo de novos alimentos, o que torna as tabelas mais completas e com valores mais precisos (ROSTAGNO, 1990; ALBINO, 1991; PUPA, 1995; AZEVEDO, 1997; e FISCHER JR., 1997).

O conhecimento do conteúdo energético dos alimentos é de suma importância para os nutricionistas, uma vez que os ingredientes são incluídos ou rejeitados nas formulações de mínimo custo, em função, prin-

\footnotetext{
1 Parte da tese apresentada pelo primeiro autor à UFV para obtenção do título de Magister Scientiae.

2 Zootecnista - Departamento de Zootecnia, UFV - 36571-000 - Viçosa, MG. Bolsista CNPq. E.mail: rvnunes@tdnet.com.br

3 Professor do Departamento de Zootecnia, UFV - 36571-000 - Viçosa, MG. Bolsista CNPq.

4 Estudante de Mestrado - UFV - 36571-000 - Viçosa, MG.
} 
cipalmente, de seu conteúdo relativo de energia (LIMA, 1996), sendo que a energia metabolizável (EM) é a melhor forma de se expressar a energia disponível para as aves. Para determinação direta dos valores de EM, utilizam-se os ensaios biológicos e de forma indireta, as equações de predição.

Devido às dificuldades de se determinar a EM dos alimentos, a utilização de tabelas e, ou, equações de predição podem ser alternativas para determinação da EMA. As equações de predição utilizam parâmetros físicos e químicos dos alimentos e podem aumentar a precisão no processo de formulação de rações, por meio da correção dos valores energéticos; conseqüentemente, a sua utilização é mais apropriada, quando a composição química dos alimentos tem grande variabilidade (ALBINO e SILVA, 1996).

Assim, torna-se importante determinar os valores

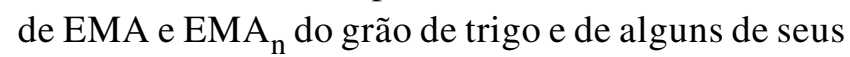
subprodutos e estabelecer equações de predição dos valores de EM, utilizando a composição químicofísica dos alimentos.

\section{Material e Métodos}

Os valores de energia metabolizável aparente (EMA) e aparente corrigida pelo balanço de nitrogênio $\left(\mathrm{EMA}_{\mathrm{n}}\right)$, de 11 alimentos, foram obtidos mediante ensaio biológico conduzido no Laboratório de Animais do Departamento de Zootecnia (DZO) da Universidade Federal de Viçosa (UFV), no período de 30/05/98 a 07/06/98, utilizando-se o método tradicional de coleta total de excretas.

A temperatura média, no interior da sala, durante a fase experimental, registrada preferencialmente às $8 \mathrm{~h}$, foi $22,0 \pm 3,2^{\circ} \mathrm{C}$ e as médias das mínimas e máximas, $19,3 \pm 2,1^{\circ} \mathrm{Ce} 24,7 \pm 1,1^{\circ} \mathrm{C}$, respectivamente.

Foram avaliados 11 alimentos, sendo quatro amostras de farelo de trigo de diferentes fornecedores e uma de farinha morena (ou farinha de segunda, terceira ou mista, obtida em uma fase intermediária no processamento do grão de trigo), farinha de trigo, gérmen de trigo, trigo-grão, triguilho (grãos pouco desenvolvidos, mal granados ou chocos resultantes de lotes cujo peso específico é menor que o mínimo exigido na moagem, ou da classificação do trigo após a eliminação de impurezas), resíduo de fábrica de biscoito e resíduo de fábrica de macarrão. As análises químicas dos alimentos foram realizadas no Laboratório de Nutrição Animal do DZO/UFV para determinar os valores de matéria seca (MS), nitrogênio $(\mathrm{N})$, extrato etéreo (EE), fibra bruta (FB), fibra em detergente neutro (FDN), fibra em detergente ácido (FDA), lignina (Lig), matéria mineral (MM), cálcio $(\mathrm{Ca})$, fósforo $(\mathrm{P})$, potássio $(\mathrm{K})$, sódio $(\mathrm{Na})$, magnésio $(\mathrm{Mg})$, cobre $(\mathrm{Cu})$, ferro $(\mathrm{Fe})$ e manganês $(\mathrm{Mn})$. A técnica utilizada para estas análises foi descrita por SILVA (1998). Determinou-se, ainda, o diâmetro geométrico médio (DGM), por intermédio da técnica adaptada de ZANOTTO e BELLAVER (1996).

Foram utilizados 480 pintos de corte, da linhagem Aviam Farms, de ambos os sexos, com 16 dias de idade e peso médio de 401,95 g. O ensaio consistiu de 11 alimentos e uma ração-referência (Tabela 1), calculada segundo ROSTAGNO et al. (1983). Cada alimento avaliado substituiu em $40 \%$ a ração-referência.

$\mathrm{O}$ delineamento experimental foi inteiramente casualizado, com 12 rações, sendo 11 alimentos-teste e uma ração-referência, quatro repetições e dez aves por unidade experimental, cinco machos e cinco fêmeas. Até o décimo sexto dia de idade, as aves receberam ração inicial para frangos de corte e ficaram alojadas em um galpão de alvenaria da Seção de Avicultura do DZO/UFV. No décimo sexto dia de idade, foram transferidas para baterias de estrutura metálica constituídas de compartimentos distribuídos em quatro andares. As baterias, em número de quatro, estavam dispostas em uma sala de $80 \mathrm{~m}^{2}$, com 4 $\mathrm{m}$ de pé direito e janelas de vidro. As aves receberam luz natural e, ou, artificial durante 24 horas. O ambiente foi climatizado com aquecedores elétricos, durante todo o período experimental, para maior conforto térmico dos animais. Durante oito dias, as aves receberam água e ração experimental à vontade, sendo três dias de adaptação e cinco para coleta total de excretas, que foram realizadas duas vezes ao dia, às 8 e $17 \mathrm{~h}$, para evitar fermentação. Para realizar a coleta e evitar perdas, utilizaram-se bandejas cobertas com plástico, colocadas sob cada andar das baterias.

Ao término do experimento, foi determinada a quantidade de ração consumida por repetição, durante os cinco dias de coleta. As excretas coletadas foram acondicionadas em sacos plásticos devidamente identificados e armazenadas em freezer até o final do período de coleta. Posteriormente, as amostras foram descongeladas, pesadas, homogeneizadas e retiradas alíquotas, para análises laboratoriais, e présecas em estufas ventiladas a $55^{\circ} \mathrm{C}$, para subseqüentes análises de matéria seca, nitrogênio e energia bruta.

Uma vez obtidos os resultados das análises laboratoriais dos alimentos, da ração-referência e das excretas, foram calculados os valores de energia 
Rev. bras. zootec.

Tabela 1 - Composição da ração referência, em porcentagem na matéria natural

Table 1 - Composition of the reference diet in percentage as fed basis

\begin{tabular}{|c|c|}
\hline $\begin{array}{l}\text { Ingredientes } \\
\text { Ingredients }\end{array}$ & $(\%)$ \\
\hline Milho & 52,08 \\
\hline \multicolumn{2}{|l|}{ Corn } \\
\hline Farelo de soja & 40,70 \\
\hline \multicolumn{2}{|l|}{ Soybean meal } \\
\hline Óleo vegetal & 3,21 \\
\hline \multicolumn{2}{|l|}{ Soybean oil } \\
\hline Fosfato bicálcico & 2,05 \\
\hline \multicolumn{2}{|l|}{ Dicalcium phosphate } \\
\hline Calcário & 0,96 \\
\hline \multicolumn{2}{|l|}{ Limestone } \\
\hline Sal & 0,40 \\
\hline \multicolumn{2}{|l|}{ Salt } \\
\hline Mistura mineral $^{1}$ & 0,07 \\
\hline \multicolumn{2}{|l|}{ Mineral mix 1} \\
\hline Mistura vitamínica ${ }^{2}$ & 0,13 \\
\hline \multicolumn{2}{|l|}{ Vitamin mix ${ }^{2}$} \\
\hline DL-Metionina $(99 \%)$ & 0,20 \\
\hline \multicolumn{2}{|l|}{ DL-Methionine } \\
\hline Cloreto de colina $(60 \%)$ & 0,06 \\
\hline \multicolumn{2}{|l|}{ Choline Chloride } \\
\hline Monensina sódica $(20 \%)$ & 0,07 \\
\hline \multicolumn{2}{|l|}{ Sodium monensin } \\
\hline Virginiamicina $(2 \%)$ & 0,06 \\
\hline \multicolumn{2}{|l|}{ Virginiamicin } \\
\hline BHT & 0,01 \\
\hline \multicolumn{2}{|l|}{ Composição calculada } \\
\hline \multicolumn{2}{|l|}{ Calculated composition } \\
\hline Proteína bruta (\%) & 23,27 \\
\hline \multicolumn{2}{|l|}{ Crude protein } \\
\hline Energia metabolizável (kcal/kg) & 3000,00 \\
\hline \multicolumn{2}{|l|}{ Metabolizable energy } \\
\hline Metionina $(\%)$ & 0,56 \\
\hline \multicolumn{2}{|l|}{ Methionine } \\
\hline Met $+\operatorname{cis}(\%)$ & 0,94 \\
\hline \multicolumn{2}{|l|}{ Meth + cys } \\
\hline $\operatorname{Lisina}(\%)$ & 1,30 \\
\hline \multicolumn{2}{|l|}{ Lysine } \\
\hline $\mathrm{Ca}(\%)$ & 1,00 \\
\hline P disponível (\%) & 0,50 \\
\hline \multicolumn{2}{|l|}{ Available P } \\
\hline $\mathrm{Na}(\%)$ & 0,21 \\
\hline \multicolumn{2}{|c|}{ 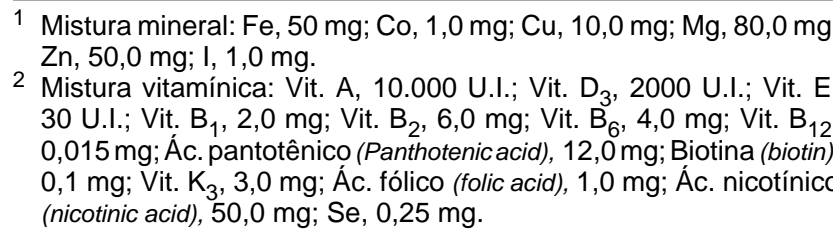 } \\
\hline
\end{tabular}

metabolizável aparente (EMA) e aparente corrigida $\left(\mathrm{EMA}_{\mathrm{n}}\right)$, por meio de equações propostas por MATTERSON et al. (1965).

Como procedimento estatístico, foram realizadas análises de regressão múltipla, para predizer os valo- res de EMA e $\mathrm{EMA}_{\mathrm{n}}$ dos alimentos-teste, utilizando-se os valores determinados de composição química (proteína bruta, matéria mineral, extrato etéreo, fibra bruta, fibra em detergente neutro e ácido, cálcio e fósforo) e o diâmetro geométrico médio (DGM).

As análises de regressão foram realizadas por regressão linear simples e múltipla, utilizando-se o Método Stepwise de Eliminação Indireta (Backward), por intermédio do Sistema de Análises Estatísticas SAEG (Universidade Federal de Viçosa, 1999).

\section{Resultados e Discussão}

Os valores de composição química e energia bruta e o diâmetro geométrico médio (DGM) e a composição em minerais dos alimentos encontram-se nas Tabelas 2 e 3, respectivamente.

Houve variação nos valores da composição química e mineral dos alimentos, quando comparados aos da literatura nacional (ROSTAGNO et al., 1983; ANFAR, 1985; EMPRESA BRASILEIRA DE PESQUISA AGROPECUÁRIA - EMBRAPA, 1991; MINISTÉRIO DA AGRICULTURA, DO ABASTECIMENTO E DA REFORMA AGRÁRIA - MAARA, 1996; e TEIXEIRA, 1998) e estrangeira (SCOTT et al., 1982; DALE et al., 1990; e NATIONAL RESEARCH COUNCIL - NRC, 1994 e 1998), havendo, inclusive, divergência entre os valores das mesmas. As diferenças encontradas podem ter sido influenciadas por vários fatores, entre eles a variação existente entre solos e climas em que são cultivados o grão de trigo, que pode ocasionar alteração em sua composição, principalmente no teor de proteína e minerais. O mesmo ocorreu em relação aos subprodutos industriais, cujo valor nutricional variou em função do processamento (ALBINO e SILVA, 1996).

Não foi possível calcular a fibra bruta dos alimentos farinha de trigo, resíduo de biscoito e resíduo de macarrão, como também os valores de fibra em detergente neutro e ácido, lignina e celulose do resíduo de biscoito, devido à formação de um gel (presença de amido), o qual impossibilitou a filtragem das amostras, mesmo tratando-as com solução de uréia a $8 \mathrm{M}$ e amilase, antes da realização das referidas análises.

Com relação aos valores de DGM, podem-se classificar os alimentos em grossos (DGM acima de $832,7 \mathrm{~mm}$ ), médios (DGM entre 375,3 e 832,7 mm) e finos (DGM menor que $375,7 \mathrm{~mm}$ ). Assim, os alimentos farelo de trigo 1 , farelo de trigo 2 , farelo de trigo 4 , trigo-grão, resíduo e biscoito, resíduo de macarrão e triguilho são classificados como médio DGM; o farelo 
NUNES et al.

Tabela 2 - Composição proximal, valores de energia bruta e DGM dos alimentos, na matéria natural ${ }^{1}$ Table 2 - Proximal composition, gross energy values and MGD of the feeds, as fed basis

\begin{tabular}{|c|c|c|c|c|c|c|c|c|c|c|}
\hline $\begin{array}{l}\text { Alimentos } \\
\text { Feedstuffs }\end{array}$ & $\begin{array}{c}\mathrm{MS} \% \\
D M\end{array}$ & $\begin{array}{c}\mathrm{PB} \% \\
C P\end{array}$ & $\begin{array}{c}\mathrm{EE} \% \\
E E\end{array}$ & $\begin{array}{c}\mathrm{FB} \% \\
C F\end{array}$ & $\begin{array}{c}\text { FDN\% } \\
N D F\end{array}$ & $\begin{array}{c}\text { FDA } \% \\
A D F\end{array}$ & $\begin{array}{l}\text { Lig\% } \\
\text { Lig } \\
\end{array}$ & $\begin{array}{c}\text { Celulose \% } \\
\text { Cellulose }\end{array}$ & $\begin{array}{c}\mathrm{DGM}(\mu \mathrm{m}) \\
\quad M G D\end{array}$ & $\begin{array}{c}\mathrm{EB}(\mathrm{kcal} / \mathrm{kg}) \\
G E \\
\end{array}$ \\
\hline $\begin{array}{l}\text { Farelo de trigo } 1 \\
\text { Wheat bran } 1\end{array}$ & 88,68 & 16,78 & 2,01 & 9,99 & 37,62 & 13,15 & 4,55 & 8,29 & 523,62 & 3701,10 \\
\hline $\begin{array}{l}\text { Farelo de trigo } 2 \\
\text { Wheat bran } 2\end{array}$ & 88,38 & 16,34 & 1,92 & 8,26 & 34,39 & 13,14 & 4,64 & 8,30 & 705,44 & 3754,55 \\
\hline $\begin{array}{l}\text { Farelo de trigo } 3 \\
\text { Wheat bran } 3\end{array}$ & 88,65 & 15,45 & 3,39 & 10,87 & 44,98 & 16,69 & 6,48 & 10,34 & 983,91 & 3968,16 \\
\hline $\begin{array}{l}\text { Farelo de trigo } 4 \\
\text { Wheat bran } 4\end{array}$ & 88,60 & 15,51 & 2,51 & 10,22 & 44,09 & 15,49 & 6,00 & 9,27 & 597,33 & 4020,88 \\
\hline $\begin{array}{l}\text { Trigo-grão } \\
\text { Wheat grain }\end{array}$ & 87,50 & 12,08 & 1,42 & 2,36 & 9,25 & 2,65 & 1,04 & 1,79 & 761,33 & 3822,59 \\
\hline $\begin{array}{l}\text { Farinha morena } \\
\text { Brown flour meal }\end{array}$ & 88,22 & 18,30 & 1,50 & 7,91 & 31,90 & 10,64 & 3,89 & 6,82 & 372,33 & 3748,28 \\
\hline $\begin{array}{l}\text { Farinha de trigo } \\
\text { Wheat flour }\end{array}$ & 86,66 & 11,96 & 2,00 & $\mathrm{ND}^{3}$ & 1,09 & 0,07 & 0,27 & 0,11 & 187,71 & 3749,26 \\
\hline $\begin{array}{l}\text { Resíduo de biscoito } \\
\text { Cookies residue }\end{array}$ & 92,42 & 8,38 & 11,89 & ND & ND & ND & ND & ND & 538,34 & 4432,04 \\
\hline $\begin{array}{l}\text { Resíduo de macarrão } \\
\text { Macaroni residue }\end{array}$ & 87,90 & 12,34 & 1,17 & ND & 2,00 & 0,60 & 0,17 & 0,24 & 549,65 & 3767,80 \\
\hline $\begin{array}{l}\text { Triguilho } \\
\text { Wheat grain residue }\end{array}$ & 88,23 & 13,33 & 2,19 & 7,30 & 18,71 & 8,85 & 2,94 & 5,52 & 512,85 & 3872,77 \\
\hline $\begin{array}{l}\text { Gérmen de trigo } \\
\text { Wheat germ }\end{array}$ & 88,32 & 28,22 & 9,56 & 2,49 & 9,32 & 3,87 & 1,14 & 2,80 & 911,68 & 4331,07 \\
\hline
\end{tabular}

Tabela 3 - Composição mineral e matéria mineral dos alimentos, na matéria natural ${ }^{1}$

Table 3 - Mineral composition and ash of the feeds, as fed basis

\begin{tabular}{|c|c|c|c|c|c|c|c|c|c|c|}
\hline $\begin{array}{l}\text { Alimentos } \\
\text { Feedstuffs }\end{array}$ & $\begin{array}{c}\mathrm{MM} \% \\
\text { Ash }\end{array}$ & $\mathrm{Ca} \%$ & $\mathrm{P} \%$ & $\mathrm{Mg} \%$ & $\mathrm{~K} \%$ & $\mathrm{Na} \%$ & Fe ppm & $\mathrm{Cu}$ ppm & Mn ppm & Zn ppm \\
\hline $\begin{array}{l}\text { Farelo de trigo } 1 \\
\text { Wheat bran } 1\end{array}$ & 4,82 & 0,16 & 0,95 & 0,41 & 1,15 & 0,04 & 305,67 & 17,85 & 88,15 & 150,31 \\
\hline $\begin{array}{l}\text { Farelo de trigo } 2 \\
\text { Wheat bran } 2\end{array}$ & 3,76 & 0,18 & 0,76 & 0,36 & 0,84 & 0,02 & 253,18 & 16,16 & 94,64 & 139,06 \\
\hline $\begin{array}{l}\text { Farelo de trigo } 3 \\
\text { Wheat bran } 3\end{array}$ & 5,56 & 0,15 & 1,11 & 0,49 & 1,20 & 0,01 & 180,03 & 16,46 & 98,54 & 169,52 \\
\hline $\begin{array}{l}\text { Farelo de trigo } 4 \\
\text { Wheat bran } 4\end{array}$ & 5,11 & 0,15 & 1,04 & 0,46 & 1,12 & 0,01 & 183,09 & 13,82 & 95,05 & 154,65 \\
\hline $\begin{array}{l}\text { Trigo-grão } \\
\text { Wheat grain }\end{array}$ & 1,45 & 0,08 & 0,30 & 0,11 & 0,34 & 0,01 & 79,71 & 4,60 & 28,32 & 56,41 \\
\hline $\begin{array}{l}\text { Farinha morena } \\
\text { Brown flour meal }\end{array}$ & 3,91 & 0,15 & 0,90 & 0,36 & 0,99 & 0,02 & 192,98 & 15,77 & 103,52 & 165,68 \\
\hline $\begin{array}{l}\text { Farinha de trigo } \\
\text { Wheat flour }\end{array}$ & 0,45 & 0,08 & 0,10 & 0,03 & 0,11 & 0,01 & 32,44 & 2,44 & 5,56 & 19,03 \\
\hline $\begin{array}{l}\text { Resíduo de biscoito } \\
\text { Cookies residue }\end{array}$ & 1,36 & 0,01 & 0,13 & 0,04 & 0,16 & 0,19 & 138,14 & 2,73 & 12,22 & 36,32 \\
\hline $\begin{array}{l}\text { Resíduo de macarrão } \\
\text { Macaroni residue }\end{array}$ & 1,36 & 0,08 & 0,13 & 0,05 & 0,18 & 0,01 & 180,26 & 2,87 & 8,20 & 34,78 \\
\hline $\begin{array}{l}\text { Triguilho } \\
\text { Wheat grain residue }\end{array}$ & 2,52 & 0,13 & 0,43 & 0,17 & 0,43 & 0,02 & 168,85 & 26,33 & 36,49 & 69,70 \\
\hline $\begin{array}{l}\text { Gérmen de trigo } \\
\text { Wheat germ }\end{array}$ & 4,01 & 0,09 & 0,84 & 0,22 & 0,79 & 0,01 & 110,62 & 2,44 & 119,92 & 204,25 \\
\hline
\end{tabular}

\footnotetext{
1 Análises realizadas no Laboratório de Nutrição Animal do Departamento de Zootecnia.
}

1 Laboratorial analyses were determined at Nutrition Animal Lab, Departament of Animal Science. 
Rev. bras. zootec.

de trigo 3 e gérmen de trigo, como grosso DGM; e a farinha morena e farinha de trigo, como fino DGM. A variação encontrada para o DGM entre os alimentos pode serem função do moinho utilizado pela fábrica, oqual nem sempre é uma característica geral dos alimentos.

Valores de energia metabolizável aparente e energia metabolizável aparente corrigida

Os valores de EMA e EMA ${ }_{n}$ e seus respectivos desvios-padrão estão representados na Tabela 4. Houve variação entre os valores de EMA e EMA, entre os alimentos estudados, devido provavelmente às variações encontradas em sua composição química e também no DGM. Os valores variaram de 1807 a 4480 e 1758 a $4339 \mathrm{kcal} / \mathrm{kg}$, para EMA e EMA, respectivamente.

Constatou-se que todos os valores de EMA foram superiores aos de $\mathrm{EMA}_{\mathrm{n}}$ em 3,80\%. BORGES et al. (1998), quando trabalharam com grão de trigo e alguns de seus subprodutos, também observaram que os valores de EMA foram superiores, em média, $5,83 \%$, quando comparados aos de EMA $\mathrm{n}$. É uma característica normal os valores de EMA, quando

Tabela 4 - Valores de energia metabolizável aparente (EMA) e aparente corrigida $\left(E M A_{n}\right)^{1}$ e seus respectivos desvios-padrão (DP)

Table 4 - Apparent metabolizable energy (AME) and corrected apparent $\left(A M E_{n}\right)$ values and its respective standard errors (SE)

\begin{tabular}{|c|c|c|c|c|}
\hline $\begin{array}{l}\text { Alimentos } \\
\text { Feedstuffs }\end{array}$ & $\begin{array}{l}\text { EMA } \\
A M E\end{array}$ & $\begin{array}{l}\text { DP } \\
S E\end{array}$ & $\begin{array}{l}\mathrm{EMA}_{\mathrm{n}} \\
A M E_{n}\end{array}$ & $\begin{array}{l}\mathrm{DP} \\
S E\end{array}$ \\
\hline Farelo de trigo 1 & 1940 & $\pm 56,73$ & 1864 & $\pm 55,83$ \\
\hline $\begin{array}{l}\text { Farelo de trigo } 2 \\
\text { Wheat bran } 2\end{array}$ & 1972 & $\pm 40,82$ & 1936 & $\pm 25,12$ \\
\hline $\begin{array}{l}\text { Farelo de trigo } 3 \\
\text { Wheat bran } 3\end{array}$ & 1807 & $\pm 83,11$ & 1758 & $\pm 86,87$ \\
\hline $\begin{array}{l}\text { Farelo de trigo } 4 \\
\text { Wheat bran } 4\end{array}$ & 1851 & $\pm 77,02$ & 1795 & $\pm 106,83$ \\
\hline $\begin{array}{l}\text { Trigo-grão } \\
\text { Wheat grain }\end{array}$ & 3593 & $\pm 169,17$ & 3457 & $\pm 156,21$ \\
\hline $\begin{array}{l}\text { Farinha morena } \\
\text { Brown flour meal }\end{array}$ & 1963 & $\pm 83,33$ & 1903 & $\pm 91,53$ \\
\hline $\begin{array}{l}\text { Farinha de trigo } \\
\text { Wheat flour }\end{array}$ & 4291 & $\pm 128,12$ & 4113 & $\pm 117,96$ \\
\hline $\begin{array}{l}\text { Resíduo de biscoito } \\
\text { Cookies residue }\end{array}$ & 4480 & $\pm 80,86$ & 4339 & $\pm 74,03$ \\
\hline $\begin{array}{l}\text { Resíduo de macarrão } \\
\text { Macaroni residue }\end{array}$ & 4105 & $\pm 22,77$ & 3943 & $\pm 13,35$ \\
\hline $\begin{array}{l}\text { Triguilho } \\
\text { Wheat grain residue }\end{array}$ & 3275 & $\pm 94,62$ & 3140 & $\pm 91,21$ \\
\hline $\begin{array}{l}\text { Gérmen de trigo } \\
\text { Wheat germ }\end{array}$ & 2963 & $\pm 72,93$ & 2813 & $\pm 75,07$ \\
\hline
\end{tabular}
1 Valores expressos em $\mathrm{kcal} / \mathrm{kg}$ de matéria seca (Values in $\mathrm{kcal} / \mathrm{kg}$ of
dry matter). determinados pelo método tradicional com pintos, serem superiores aos de $\mathrm{EMA}_{\mathrm{n}}$, o que é caracterizado pela retenção de nitrogênio pelas aves. De acordo com COELHO (1983), em níveis normais de consumo, as perdas de energia fecal metabólica (EFm) e de energia urinária endógena (EUe) são pequenas em relação à excreção de energia proveniente do alimento e têm pouca influência nos valores de EMA e EMA obtidos pelo método tradicional.

Os valores de EMA e EMA ${ }_{n}$, respectivamente, para a farinha morena, encontraram-se próximos à variação entre os diferentes farelos de trigo, os quais variaram de 1807 a 1972; os valores da farinha morena foram de 1758 a $1936 \mathrm{kcal} / \mathrm{kg}$. BORGES et al. (1998) encontraram, para os diversos tipos de farelos de trigo, variação de 1686 a 2028 e 1557 a $1911 \mathrm{kcal} / \mathrm{kg}$ de EMA e EMA , respectivamente. Já TEIXEIRA (1998) encontrou valor para EMA de $1526 \mathrm{kcal} / \mathrm{kg}$, para o farelo de trigo. Com relação à literatura estrangeira, os valores de EMA $_{n}$ encontrados por SCOTT et al. (1982) e pelo NRC (1994) foram de $1300 \mathrm{kcal} / \mathrm{kg}$ para o farelo, entretanto, no AERC (1989), o valor de EMA $_{n}$ foi de $1870 \mathrm{kcal} / \mathrm{kg}$.

Os alimentos com maiores valores de EMA e $\mathrm{EMA}_{\mathrm{n}}$ foram resíduo de biscoito, farinha de trigo, resíduo de macarrão, trigo-grão, triguilho e gérmen de trigo, tendo variação de 4480 a $2963 \mathrm{kcal} / \mathrm{kg}$ para EMA e de 4339 a 2813 kcal/kg para EMA . Para a farinha de trigo, BORGES et al. (1998) citaram valores 6,06 a 5,52\% menores para EMA e EMA ${ }_{n}$, respectivamente, e no AERC (1989) o valor citado foi menor em $18,5 \%$ para $\mathrm{EMA}_{\mathrm{n}}$, em relação aos deste trabalho. Para o grão de trigo, os valores de EMA $_{n}$ que mais se aproximaram foram os encontrados por SCOTT et al. (1982) e pelo AERC (1989), 3250 e $3530 \mathrm{kcal} / \mathrm{kg}$, respectivamente.

Para o gérmen de trigo, os valores de 2963 e $2813 \mathrm{kcal} / \mathrm{kg}$ para EMA e EMA ${ }_{\mathrm{n}}$, respectivamente, estão próximos aos encontrados por outros autores; SCOTT et al. (1982) encontraram $2700 \mathrm{kcal} / \mathrm{kg}$ para EMA e BORGES et al. (1998), valores de EMA e $\mathrm{EMA}_{\mathrm{n}}$ de 3006 e $2758 \mathrm{kcal} / \mathrm{kg}$, respectivamente. TEIXEIRA (1998) encontrou valor $3086 \mathrm{kcal} / \mathrm{kg}$ para EMA. O triguilho apresentou valor superior em 18,66\% para EMA, quando comparado ao valor citado pela EMBRAPA (1991).

Com relação aos resíduos estudados (biscoito e macarrão), os valores de $\mathrm{EMA}_{\mathrm{n}}$ foram comparados com os valores de literatura obtidos para resíduos de panificação, sendo que o valor de EMA $\mathrm{n}_{\mathrm{n}}$ para resíduo 
de panificação, citado no AERC (1989), foi $3690 \mathrm{kcal} / \mathrm{kg}$ e no NRC (1994), $3862 \mathrm{kcal} / \mathrm{kg}$, valores inferiores aos determinados neste trabalho, $4339 \mathrm{e}$ $3943 \mathrm{kcal} / \mathrm{kg}$, respectivamente, para os resíduos de biscoito e macarrão.

As diferenças encontradas nos valores de EMA e $\mathrm{EMA}_{\mathrm{n}}$ podem ser utilizadas para classificar os alimentos em alta e baixa energia, quando o valor de EMA ficar, respectivamente, acima e abaixo de $2500 \mathrm{kcal} / \mathrm{kg}$. Esta classificação pode estar relacionada com a composição química dos alimentos, pois os alimentos que apresentam maior concentração de amido têm maiores valores para EMA; em contrapartida, os alimentos com maior teor de polissacarídeos não-amiláceos têm menores valores de EMA. Segundo o INSTITUT NATIONAL DE LA RECHERCH AGRONOMIQUE - INRA (1984), a farinha de trigo, o trigo-grão, o triguilho e o gérmen de trigo contêm 48,0 a 58,3; 53,5; 40,0; e $17,6 \%$ de amido, respectivamente, enquanto, nos diferentes farelos de trigo, podem variar de 16,5 a $19,4 \%$ de amido entre farelos grossos e finos.

Equações de predição dos valores energéticos dos alimentos

As equações de regressão múltipla calculadas para estimar os valores de EMA e $\mathrm{EMA}_{\mathrm{n}}$ dos alimentos encontram-se, respectivamente, nas Tabelas
5 e 6. Foram utilizadas as variáveis: proteína bruta $(\mathrm{PB})$, fibra bruta $(\mathrm{FB})$, extrato etéreo (EE), matéria mineral (MM), fibra em detergente neutro (FDN), fibra em detergente ácido (FDA), cálcio (Ca), fósforo (P) e diâmetro geométrico médio (DGM).

Entre todos os parâmetros utilizados para determinar as equações de predição, o EE foi o único que teve correlação positiva com os valores de EMA e EMA $_{n}$, porém com baixa correlação $( \pm 32 \%)$. Para as demais variáveis independentes, as que apresentaram maior correlação com os valores de EMA e $\mathrm{EMA}_{\mathrm{n}}$, porém correlação negativa, foram FDN $( \pm 95 \%), \mathrm{MM}( \pm 94 \%), \mathrm{FB}( \pm 93 \%), \mathrm{P}( \pm 93 \%), \mathrm{Ca}$ $( \pm 90 \%)$, FDA $( \pm 84 \%), \mathrm{PB}( \pm 49)$ e DGM $( \pm 35 \%)$. Para se obterem equações com maior precisão, foi utilizado o teste $\mathrm{T}$, com significância de $5 \%$ de probabilidade, isoladamente dentro de cada variável que compõe a equação.

As equações foram calculadas pelo Método Stepwise de Eliminação Indireta (Backward), o qual fornece a contribuição de cada variável dentro da análise de regressão múltipla. Deve-se observar que este método mostra a equação que melhor representa o fenômeno estudado e, por meio da retirada de uma variável por vez, pela significância do teste $T$, é excluída a variável que menos contribui na determinação do valor de energia, até a obtenção de uma

Tabela 5 - Equações de predição dos valores de EMA, a partir da composição química ${ }^{1}$ dos alimentos Table 5 - Prediction equation of the AME values, in the chemical composition ${ }^{1}$ of the feedstuffs

\begin{tabular}{|c|c|c|c|c|c|c|c|c|c|c|}
\hline \multirow{3}{*}{$\begin{array}{l}\text { Constante } \\
\text { Constant }\end{array}$} & \multicolumn{9}{|c|}{$\begin{array}{c}\text { Equações dos valores de EMA } \\
\text { Equations of the AME values }\end{array}$} & \multirow{3}{*}{$\mathrm{R}^{2}$} \\
\hline & PB & $\mathrm{FB}$ & FDN & FDA & $\mathrm{EE}$ & $\mathrm{MM}$ & $\mathrm{Ca}$ & $\mathrm{P}$ & DGM & \\
\hline & $C P$ & $C F$ & $N D F$ & $A D F$ & & & & & $M G D$ & \\
\hline$+5011,96$ & $-64,46$ & $+33,96$ & $-73,50$ & $+18,39$ & & & & $+451,48$ & $+0,19$ & 0,99 \\
\hline$+5054,52$ & $-59,87$ & & $-62,95$ & $+16,68$ & & & & $+398,68$ & & 0,98 \\
\hline$+4907,94$ & $-46,57$ & & $-56,10$ & $+17,95$ & & & & & & 0,98 \\
\hline$+4910,03$ & $-47,82$ & & $-47,77$ & & & & & & & 0,98 \\
\hline$+4650,21$ & & & $-19,96$ & & & & $-5054,42$ & $-886,39$ & & 0,97 \\
\hline$+4681,96$ & $-27,32$ & $-92,95$ & & & $+41,19$ & $-247,80$ & & & & 0,96 \\
\hline$+5165,63$ & & & & & & $-322,97$ & $-8452,55$ & & & 0,96 \\
\hline$+4392,97$ & & $-46,79$ & & & $+51,49$ & $-392,65$ & & & & 0,95 \\
\hline$+4390,38$ & & & & & $+70,22$ & $-494,38$ & & & & 0,94 \\
\hline$+4291,75$ & & & $-33,00$ & & & & & $-863,06$ & & 0,95 \\
\hline$+4871,93$ & $-36,32$ & $-151,18$ & & & & $-109,29$ & & & & 0,94 \\
\hline$+4929,29$ & $-49,57$ & $-185,81$ & & & & & & & & 0,94 \\
\hline$+4159,83$ & & & $-51,23$ & & & & & & & 0,92 \\
\hline$+4705,61$ & & & & & & $-503,74$ & & & & 0,87 \\
\hline$+4151,75$ & & $-199,94$ & & & & & & & & 0,87 \\
\hline
\end{tabular}

${ }^{1}$ Valores expressos na matéria seca: $\mathrm{PB}=$ proteína bruta $(\%) ; \mathrm{EE}=$ extrato etéreo $(\%) ; \mathrm{FB}=$ fibra bruta $(\%) ; \mathrm{FDN}=$ fibra em detergente neutro $(\%)$; $\mathrm{FDA}=$ fibra em detergente ácido (\%); $\mathrm{MM}=$ matéria mineral $(\%) ; \mathrm{Ca}=$ cálcio $(\%) ; \mathrm{P}=$ fósforo $(\%) ; \mathrm{e} \mathrm{DGM}=$ diâmetro médio geométrico $(\mu \mathrm{m})$.

1 Values in dry matter basis: $C P=$ crude protein (\%); $E E=$ ether extract $(\%) ; C F=$ crude fiber $(\%) ; N D F=$ neutral detergent fiber $(\%) ; A D F=$ acid detergent fiber $(\%)$; $M M=\operatorname{ash}(\%) ; C a=$ calcium (\%); $P=$ phosphorus $(\%) ; M G D=$ medium geometric diameter $(\mu \mathrm{m})$. 
Rev. bras. zootec.

Tabela 6 - Equações de predição dos valores de $\mathrm{EMA}_{\mathrm{n}}$, a partir da composição química ${ }^{1}$ dos alimentos

Table 6 - Prediction equations of the $A M E_{n}$ values, in the chemical composition ${ }^{1}$ of the feedstuffs

\begin{tabular}{|c|c|c|c|c|c|c|c|c|c|c|}
\hline \multirow{3}{*}{$\begin{array}{l}\text { Constante } \\
\text { Constant }\end{array}$} & \multicolumn{10}{|c|}{$\begin{array}{l}\text { Equações dos valores de } \mathrm{EMA}_{\mathrm{n}} \\
\text { Equation of the } A M E_{n} \text { values }\end{array}$} \\
\hline & PB & FB & FDN & FDA & $\mathrm{EE}$ & MM & $\mathrm{Ca}$ & $\mathrm{P}$ & DGM & $\mathrm{R}^{2}$ \\
\hline & $C P$ & $C F$ & $N D F$ & $A D F$ & & & & & $M G D$ & \\
\hline$+4854,72$ & $-64,13$ & & $-61,02$ & $+15,88$ & & & & $+424,30$ & $+0,18$ & 0,99 \\
\hline$+4898,07$ & $-60,52$ & & $-59,88$ & $+15,35$ & & & & $+398,63$ & & 0,99 \\
\hline$+4752,12$ & $-47,23$ & & $-53,01$ & $+16,57$ & & & & & & 0,98 \\
\hline$+4754,02$ & $-48,38$ & & $-45,32$ & & & & & & & 0,98 \\
\hline$+4476,84$ & & & $-17,42$ & & & & $-4994,13$ & $-895,97$ & & 0,96 \\
\hline$+4947,88$ & & & & & & $-308,88$ & $-8022,85$ & & & 0,96 \\
\hline$+4536,71$ & $-29,55$ & $-89,17$ & & & $+40,30$ & $-231,43$ & & & & 0,96 \\
\hline$+4224,44$ & & $-39,30$ & & & $+51,39$ & $-388,02$ & & & & 0,95 \\
\hline$+4689,44$ & & & & & & & $-7978,04$ & $-1268,00$ & & 0,95 \\
\hline$+4129,41$ & & & $-30,42$ & & & & & $-873,94$ & & 0,95 \\
\hline$+4772,55$ & $-49,96$ & $-176,55$ & & & & & & & & 0,94 \\
\hline$+4222,41$ & & & & & $+67,10$ & $-476,46$ & & & & 0,94 \\
\hline$+3994,87$ & & & $-48,82$ & & & & & & & 0,91 \\
\hline$+4523,97$ & & & & & & $-482,50$ & & & & 0,88 \\
\hline$+3988,90$ & & $-190,80$ & & & & & & & & 0,87 \\
\hline
\end{tabular}

equação de apenas uma variável.

Os coeficientes de determinação das equações de regressão $\left(\mathrm{R}^{2}\right)$ variaram de 0,99 a 0,87 , para os valores de EMA e EMA ${ }_{n}$, utilizando-se equações com seis a uma variável. Entretanto, as equações com duas a quatro variáveis podem ser utilizadas com maior facilidade, pois as análises laboratoriais de poucas variáveis são realizadas em menor tempo e de forma mais econômica, obtendo-se, assim, resultados confiáveis, com $\mathrm{R}^{2}$ superior a 0,94 , tanto para os valores de EMA, quanto para os de EMA $_{n}$.

Foi observado que todos os efeitos são significativos dentro de cada variável em sua respectiva equação, em nível de 5\% de probabilidade, pelo teste $\mathrm{T}$, evidenciando que as equações são as que melhor predizem os valores de EMA e EMA . $_{n}$.

Entre as possíveis combinações com duas variáveis, a que proporcionou melhor $\mathrm{R}^{2}$ foi aquela composta pelas variáveis PB e FDN, com $R^{2}$ igual a 0,98 , para os valores de EMA e EMA ${ }_{n}$, sendo a equação que melhor se ajustou na predição dos valores de EMA e EMA $A_{n}$. As equações obtidas da combinação de variáveis duas a duas, geralmente, são as que apresentam melhores ajustes na predição dos valores de EM (PESTI et al., 1986; DOLZ e DE BLAS,
1992; e DUDLEY-CASH, 1994). Para uma variável, a FDN foi a que proporcionou melhor valor de $\mathrm{R}^{2}$, 0,92 e 0,91, para EMA e EMA ${ }_{n}$, respectivamente.

Considerando-se a rapidez e o custo das análises, podem-se obter bons resultados utilizando as variáveis $\mathrm{MM}$ e $\mathrm{EE}\left(\mathrm{R}^{2}=0,94\right)$, ou $\mathrm{PB}$ e FDN $\left(R^{2}=0,98\right)$, para predição dos valores de EMA e EMA $A_{n}$.

$\mathrm{Na}$ Tabela 7, estão apresentados os valores de EMA $_{n}$ dos alimentos obtidos no ensaio de metabolismo, os estimados por três equações com as variáveis independentes proteína bruta (PB), fibra em detergente neutro (FDN), matéria mineral (MM) e extrato etéreo (EE), a somatória ao quadrado das diferenças dos valores estimados e observados e a média das diferenças entre os valores estimados e observados.

Ao analisar a média dos desvios, observou-se que a diferença se eleva, à medida que o $\mathrm{R}^{2}$ diminui. Assim, com $\mathrm{R}^{2}$ de 0,98 , tem-se uma média das diferenças de 15.736; com $\mathrm{R}^{2}$ de 0,94 , esta média eleva-se para 47.579 e, com $R^{2}$ de 0,91 , para 75.987 . Com relação à média das diferenças, a equação composta pelas variáveis $\mathrm{PB}$ e $\mathrm{FDN}\left(\mathrm{R}^{2}=0,98\right)$ foi a que melhor estimou os valores de EMA $_{n}$.

Para o resíduo de biscoito, somente foi possível 
Tabela 7 - Estimativa dos valores de $\mathrm{EMA}_{\mathrm{n}}$ por meio das equações de predição Table 7 - Estimate of the $A M E_{n}$ values by the prediction equations

\begin{tabular}{|c|c|c|c|c|}
\hline \multirow[b]{2}{*}{ Alimentos } & \multicolumn{3}{|c|}{$\begin{array}{l}\text { EMA }_{\mathrm{n}} 1=4754,02-48,38 * \mathrm{~PB}-45,32 * \mathrm{FDN} \\
A M E_{n} 1=4754.02-48.38 * C P-45.32 * N D F \\
\mathrm{EMA}_{\mathrm{n}} 2=4222,41+67,10 * \mathrm{EE}-473,46 * \mathrm{MM} \\
A M E_{n} 2=4222.41+67,10 * E E-473.46 * M M \\
\mathrm{EMA}_{\mathrm{n}} 3=3994,87-48,82 * \mathrm{FDN} \\
A M E_{n} 3=3994.87-48.82 * N D F\end{array}$} & \multirow{3}{*}{$\begin{array}{c}\left(\mathrm{R}^{2}=0,98\right) \\
\left(R^{2}=0,98\right) \\
\left(\mathrm{R}^{2}=0,94\right) \\
\left(R^{2}=0,94\right) \\
\left(\mathrm{R}^{2}=0,91\right) \\
\left(R^{2}=0,91\right) \\
\text { EMA }_{\mathrm{n}} 3 \\
\text { est. }\end{array}$} \\
\hline & $\mathrm{EMA}_{\mathrm{n}}$ & $\mathrm{EMA}_{\mathrm{n}} 1$ & $\mathrm{EMA}_{\mathrm{n}} 2$ & \\
\hline Feedstuffs & obs. $^{1}$ & est. $^{2}$ & est. & \\
\hline $\begin{array}{l}\text { Farelo de trigo } 1 \\
\text { Wheat bran } 1\end{array}$ & 1864 & 1916 & 1799 & 1924 \\
\hline $\begin{array}{l}\text { Farelo de trigo } 2 \\
\text { Wheat bran } 2\end{array}$ & 1936 & 2096 & 2356 & 2095 \\
\hline $\begin{array}{l}\text { Farelo de trigo } 3 \\
\text { Wheat bran } 3\end{array}$ & 1758 & 1611 & 1510 & 1518 \\
\hline $\begin{array}{l}\text { Farelo de trigo } 4 \\
\text { Wheat bran } 4\end{array}$ & 1795 & 1652 & 1680 & 1566 \\
\hline $\begin{array}{l}\text { Trigo-grão } \\
\text { Whea grain }\end{array}$ & 3457 & 3607 & 3545 & 3479 \\
\hline $\begin{array}{l}\text { Farinha morena } \\
\text { Brown flour meal }\end{array}$ & 1903 & 2112 & 2239 & 2230 \\
\hline $\begin{array}{l}\text { Farinha de trigo } \\
\text { Wheat flour }\end{array}$ & 4113 & 4029 & 4131 & 3933 \\
\hline $\begin{array}{l}\text { Resíduo de biscoito } \\
\text { Cookies residue }\end{array}$ & 4339 & - & 4390 & - \\
\hline $\begin{array}{l}\text { Resíduo de macarrão } \\
\text { Macaroni residue }\end{array}$ & 3943 & 3971 & 3578 & 3884 \\
\hline $\begin{array}{l}\text { Wheat grain residue } \\
\text { Gérmen de trigo }\end{array}$ & 2813 & 2730 & 2799 & 3480 \\
\hline $\begin{array}{l}\text { Wheat germ } \\
\text { Média }\end{array}$ & 2824 & 2679 & 2824 & 2707 \\
\hline $\begin{array}{l}\text { Mean } \\
{\text { Siii } \mathrm{d}^{2}} \\
\text { Média }^{4} \mathrm{~d}^{2}\end{array}$ & & $\begin{array}{c}157.356 \\
15.736\end{array}$ & $\begin{array}{c}523.365 \\
47.579\end{array}$ & $\begin{array}{l}759.866 \\
75.987\end{array}$ \\
\hline $\begin{array}{l}1 \text { Energia metabolizável a } \\
2 \text { Energia metabolizável a } \\
3 \text { Somatória ao quadrado } \\
4 \text { Média das diferenças. } \\
1 \text { Corrected apparent metabo } \\
2 \text { Corrected apparent metabo } \\
3 \text { Amount to square of the diff } \\
4 \text { Amean of the differences }\end{array}$ & $\begin{array}{l}\text { orrigida o } \\
\text { orrigida e } \\
\text { enças en } \\
\text { rgy observe } \\
\text { rgy estimat } \\
\text { nong the es }\end{array}$ & $\begin{array}{l}\text { kcal } / \mathrm{kg} \mathrm{d} \\
\text { de matéria } \\
\text { ados e obs } \\
\text { dry matter. } \\
\text { er. } \\
\text { alues. }\end{array}$ & & \\
\hline
\end{tabular}

estimar o valor de $\mathrm{EMA}_{\mathrm{n}}$ por intermédio da equação que continha as variáveis $\mathrm{MM}$ e EE, pois as equações que continham FDN impossibilitaram a sua predição, sendo que esta variável não foi determinada na análise laboratorial.

\section{Conclusões}

Os valores de EMA e EMA $\mathrm{n}_{\mathrm{n}}$ médios dos quatro farelos de trigo, expressos em kcal $/ \mathrm{kg}$ de matéria seca, foram, respectivamente, 1893 e 1838 e 1963 e 1903 para farinha morena. Para os demais alimentos, os valores de
EMA e EMA ${ }_{\mathrm{n}}$, expressos em $\mathrm{kcal} / \mathrm{kg}$ de matéria seca, foram, respectivamente, 3593 e 3457 para o trigo-grão; 3275 e 3140 para o triguilho; 4291 e 4113 para a farinha de trigo; 4480 e 4339 para o resíduo de biscoito; 4105 e 3943 para o resíduo de macarrão; e 2963 e 2813 para o gérmen de trigo. As equações de predição que melhor estimaram os valores de EMA e EMA $\mathrm{E}_{\mathrm{n}}$ foram as que continham as variáveis $\mathrm{PB}$ e FDN, as quais melhor estimaram os valores energéticos dos alimentos. As equações obtidas foram: $\mathrm{EMA}=4910,03-47,82 * \mathrm{~PB}$ $47,77 *$ FDN $\left(R^{2}=98 \%\right)$ e EMA $_{n}=4754,02-48,38 * \mathrm{~PB}$ - 45,32*FDN $\left(R^{2}=98 \%\right)$. 


\section{Referências Bibliográficas}

ALBINO, L.F.T. Sistemas de avaliação nutricional de alimentos e suas aplicações na formulação de rações para frangos de corte. Viçosa, UFV, 1991. 141p. Tese (Doutorado em Zootecnia) - Universidade Federal de Viçosa, 1991.

ALBINO, L.F.T., SILVA, M.A. Valores nutritivos de alimentos para aves e suínos determinados no Brasil. In: SIMPÓSIO INTERNACIONAL SOBREEXIGÊNCIAS NUTRICIONAIS DE AVES E SUÍNOS. Viçosa, 1996. Anais... Viçosa: UFV, 1996. p.303-318.

AERC. 1989. European table of energy values for poultry feedstuffs. 3.ed. 84p.

ANFAR. 1985. Matérias-primas para alimentação animal padrão. ANFAR. 4.ed. 65p.

AZEVEDO, D.M.S. Fatores que afetam os valores de energia metabolizável da farinha de carne e ossos para aves. Viçosa, UFV, 1996. 89p. Dissertação (Mestrado em Zootecnia) Universidade Federal de Viçosa, 1997.

BORGES, F.M.O., ROSTAGNO, H.S., BAIÃO, N.C. et al. Avaliação de métodos para estimar energia metabolizável em alimentos para aves. In: REUNIÃO Da SOCIEDADE BRASILEIRA DE ZOOTECNIA, 35, Botucatu, 1998. Anais... Botucatu: FMVZ-UNESP, 1998. p.386-388.

COELHO, M.G.R. Valores energéticos e de triptofano metabolizável de alimentos para aves, utilizando duas metodologias. Viçosa, UFV, 1983.77p. Dissertação (Mestrado em Zootecnia) - Universidade Federal de Viçosa, 1983.

COON, C.N. Optimizing ingrediente utilization trhough a better understanding of aminoacid biovailability. In: TECHNICAL SYMPOSIA, Aruba, 1991. Proceedings... Aruba: NOVUS INTERNATIONAL, 1991. p.11-40.

DALE, N.M., PESTI, G.M., ROGERS, S.R. 1990. True metabolizable energy of dried bakery product. Poult. Sci., 69(1):72-75.

DOLZ, S., DE BLAS, C. 1992. Metabolizable energy of meat and bone meal from Spanish rendering plants as influenced by level of substitution and method of determination. Poult. Sci., 71(2):316-322.

DUDLEY-CASH, W.A. 1994. Metabolizable energy value of poultry offal meal can vary widely. Feedstuffs. 66(1):11.

EMPRESA BRASILEIRA DE PESQUISA AGROPECUÁRIA EMBRAPA. 1991. Centro Nacional de Pesquisa de Suínos e Aves (CNPSA) - CNPSA. Tabela de composição química e valores energéticos de alimentos para suínos e aves. 3.ed. Concórdia-SC, EMBRAPA-CNPSA. 97p. [Documento 19].

FISCHER JR., A.A. Valores de energia metabolizável e de aminoácidos digestíveis de alguns alimentos para aves. Viçosa, UFV, 1997. 55p. Dissertação (Mestrado em Zootecnia) - Universidade Federal de Viçosa, 1997.

INSTITUT NATIONAL DE LA RECHERCH AGRONOMIQUE - INRA. 1984. Alimentacion de los Conejos. In: Alimentation des Animaux Monogastriques. Institut National de la Recherch Agronomique. Porc, Lapin, Volailles. France. p.95-243.
LIMA, I.L. Níveis nutricionais utilizados nas rações pela indústria avícola. In: SIMPÓSIO INTERNACIONAL SOBRE EXIGÊNCIAS NUTRICIONAIS DE AVES E SUÍNOS. Viçosa, 1996. Anais... Viçosa: UFV, 1996. p.389-402.

MATTERSON, L.D., POTTER, L.M., STUTZ, M.W. et al. 1965. The metabolizable energy of feed ingredients for chickens. University of Connecticut Storrs. Agric. Exp. Stat. Res. Rep., 11:11.

NATIONAL RESEARCH COUNCIL - NRC. 1994. Nutrients requirements of poultry, 9 .ed. Washington: National Academy of Sciences. 155p.

NATIONAL RESEARCH COUNCIL - NRC. 1998. Nutrients requirements of swine, 10.ed. Washington: National Academy of Sciences. 189p.

MINISTÉRIO DA AGRICULTURA, DO ABASTECIMENTO E DA REFORMA AGRÁRIA - MAARA. 1996. Normas e padrões de nutrição e alimentação animal. Revisão 96. 145p.

PESTI, G.M., FAUST, L.O., FULLER, H.L. et al. 1986. Nutritive value of poultry by-product meal. 1. Metabolizable energy values as influenced by method of determination and level substitution. Poult. Sci., 65(12):2258-2267.

PUPA, J.M.R. Rações para frangos de corte formuladas com valores de aminoácidos digestíveis verdadeiros, determinados com galos cecectomizados. Viçosa, UFV, 1995. 63p. Dissertação (Mestrado em Zootecnia) - Universidade Federal de Viçosa, 1995.

ROSTAGNO, H.S. Valores de composição de alimentos e exigências nutricionais utilizados na formulação de rações para aves. In: REUNIÃO ANUAL DA SOCIEDADE BRASILEIRA DE ZOOTECNIA, 27, Piracicaba, 1990. Anais... Piracicaba: FEALQ, 1990. p.11-30.

ROSTAGNO, H.S., SILVA, D.J., COSTA, P.M.A. et al. 1983. Composição de alimentos e exigências nutricionais de aves e suínos (Tabelas brasileiras). Viçosa: UFV. 59p.

SCOTT, M.L., NESHEIM, M.C., YOUNG, R.J. 1982. Nutrition of the chicken. 3.ed. Ithaca, NY. 562p.

TEIXEIRA, A.S. 1998. Tabelas de composição dos alimentos $e$ exigências Nutricionais. Alimentos e alimentação dos animais. UFLA/FAEPE. v.2. p.98.

UNIVERSIDADE FEDERAL DE VIÇOSA - UFV. 1999. Manual de utilização do programa SAEG (Sistema para Análise Estatísticas e Genéticas). Viçosa: UFV. 59p.

ZANOTTO, D.L., BELLAVER, C. 1996. Método de determinação da granulometria de ingredientes para uso em rações de suínos e aves. Comunicado Técnico. EMBRAPA suínos e aves. p.1-5.

Recebido em: 08/02/00 Aceito em: 04/01/01 